\title{
Feature Extraction and Classification of EEG Signals for Rapid P300 Mind Spelling
}

\author{
Adrien Combaz ${ }^{1}$, Nikolay V. Manyakov ${ }^{1}$, Nikolay Chumerin ${ }^{1}$, Johan A. K. Suykens ${ }^{2}$, Marc M. Van \\ Hulle $^{1}$ \\ ${ }^{1}$ Laboratorium voor Neuro-en Psychofysiologie, Katholieke Universiteit Leuven BELGIUM \\ ${ }^{2}$ ESAT/SCD-SISTA, Katholieke Universiteit Leuven BELGIUM \\ \{Adrien.Combaz, NikolayV.Manyakov,Nikolay.Chumerin,Marc.VanHulle\}@med.kuleuven.be, \\ Johan.Suykens@esat.kuleuven.be
}

\begin{abstract}
The Mind Speller is a Brain-Computer Interface which enables subjects to spell text on a computer screen by detecting P300 Event-Related Potentials in their electroencephalograms. This BCI application is of particular interest for disabled patients who have lost all means of verbal and motor communication. We report on the implementation of a feature extraction procedure on a new ultra low-power 8-channel wireless EEG device. The feature extraction procedure is based on downsampled EEG signal epochs, the Student's t-statistic of the Continuous Wavelet Transform, and the Common Spatial Pattern technique. For classification, we use a linear Least-Squares Support Vector Machine. The results show that subjects are potentially able to communicate a character in less than ten seconds with an accuracy of $94.5 \%$, which is more than twice as fast as the state of the art. In addition since our EEG device is wireless it offers an increased comfort to the subject.
\end{abstract}

\section{Introduction}

Brain Computer Interfaces (BCIs) are aimed at creating a direct communication pathway between the brain and an external device, bypassing the need for an embodiment. In the last few years, research in the field of BCI has witnessed a spectacular development (see [1], [2]) and is nowadays regarded as one of the most successful applications of the neurosciences. Indeed, such systems can provide a significant improvement of the quality of life of neurologically impaired patients suffering of pathologies such as amyotrophic lateral sclerosis, brain stroke, brain/spinal cord injury, etc...

In invasive BCIs, a micro-electrode array is implanted in the brain (mainly in the motor or premotor frontal areas or into the parietal cortex [3]), while in non-invasive BCIs, mainly electroencephalograms (EEGs) are recorded from the scalp. Three types of EEG-based BCIs can be distinguished. The first one is based on the SteadyState Visually Evoked Potential (SSVEP, [4]): a subject focuses on a visual stimulus flickering at a frequency $f$. This frequency $f$ and its harmonics $2 f$ and $3 f$ are detected in the Fourier transform of the EEG. By presenting the subject simultaneously several stimuli with different flickering frequencies, and associating each stimulus to some pre-defined instructions; subjects were able to navigate a small car on a computer screen [5], or to "mentally" dial a phone number [6]. The second type of EEG-based BCIs relies on the detection of mental tasks (imagination of right/left hand movements, subtraction, word association, etc...) which are detected through slow cortical potentials (SCP) [7], readiness potential [8] and event-related desynchronization (ERD) [9].

The third group, the one this article is focusing on, is based on the "oddball" evoked potential. An EventRelated Potential (ERP) is a stereotyped electrophysiological response to an external or internal stimulus [10]. One of the most known and studied ERP is the P300 ERP. It appears when a subject performs the classification of two types of events, one of which is only rarely presented (oddball paradigm). The rare event will elicit in the EEG of the subject an ERP with an enhanced positive-going component at a latency of about $300 \mathrm{~ms}$ [11]. The most popular P300-based BCI is the P300 Mind Speller [12] which allows a subject to spell words by focusing on the corresponding characters shown in a matrix display (Fig.1), while the rows and columns of the matrix are consecutively and randomly highlighted. Highlighting of a row or column containing the target character will elicit a P300 ERP and, by detecting this $\mathrm{ERP}$, the $\mathrm{BCI}$ is able to retrieve the character the subject has in mind. 

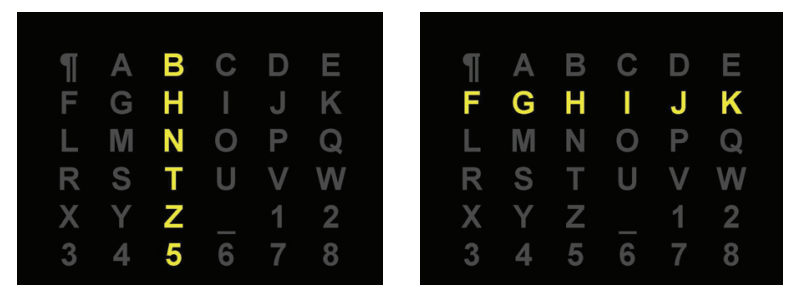

Figure 1. User display for the P300 MindSpeller BCI

The challenge is to detect this P300 ERP in the very low signal-to-noise ratio EEG. Indeed the recorded signals are a superposition of the ongoing activity in the brain and the noise generated by the hardware, which makes the P300 component generally not distinguishable in a single trial. A common practice, to enhance the signal-to-noise ratio, is to average over several trials. By averaging the recordings, the brain activity that is timelocked to the stimulus onset will be extracted as the ERP, while the activity that is not time-locked will be averaged out (Fig.2). The drawback of averaging is that it leads to a dramatic increase of the time needed to communicate a character, hence the continued effort to develop new features and classifiers to reduce the required number of trials.
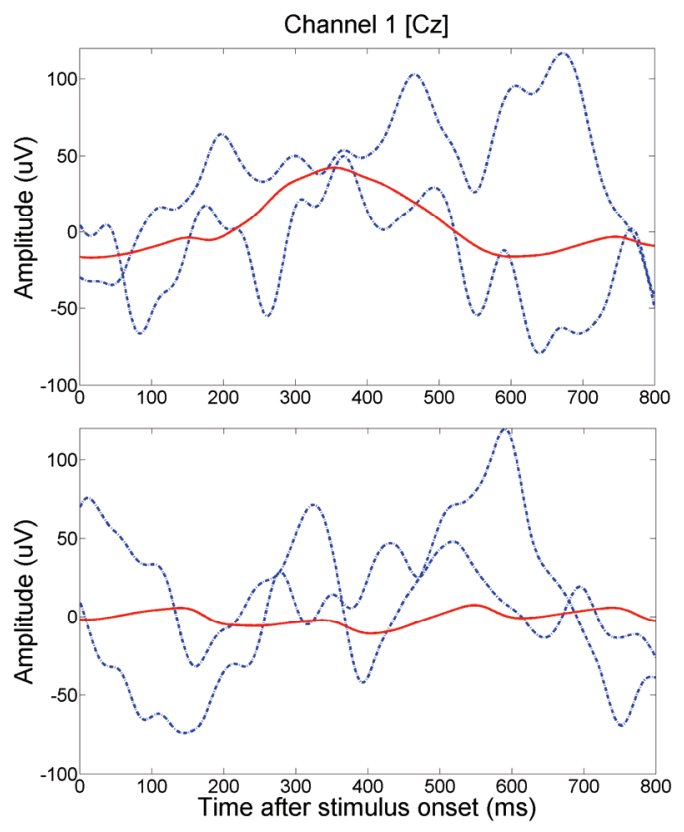

Figure 2. Responses to target (top) and nontarget (bottom) stimuli at electrode Cz. Single trials (dashed) and averages (full). Signals are filtered between 0.5 and $15 \mathrm{~Hz}$.
This article combines different feature extraction techniques with the aim of reducing the number of required trials: 1) a downsampled, filtered EEG signal, 2) a time-frequency analysis (Continuous Wavelet Transform, [13]), and 3) the Common Spatial Pattern technique [14]. The classifier used is a linear LeastSquares Support Vector Machine [15]. The results show that, by averaging the signals over only four trials, subjects are potentially able to communicate a character with an average accuracy of $94.5 \%$, which is more than twice as fast as the state of the art.

\section{Material and experiment}

\subsection{Acquisition procedure}

The EEG recordings were performed using a prototype of an ultra low-power 8-channel wireless EEG system, which consists of two parts: an amplifier coupled with a wireless transmitter and a USB stick receiver (Fig.3). The data is transmitted with a sampling frequency of $1000 \mathrm{~Hz}$ for each channel. The prototype was developed by the Interuniversity Microelectronics Center (IMEC, [16]). We used a brain-cap with large filling holes and sockets for $\mathrm{Ag} / \mathrm{AgCl}$ ring electrodes.
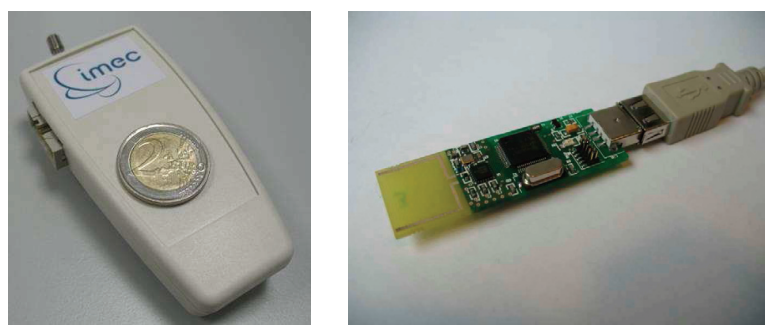

\section{Figure 3: Wireless 8 channels EEG device. Left: amplifier and transmitter. Right: USB stick receiver.}

The recordings were collected from eight electrodes in the occipital and parietal areas, namely in positions $\mathrm{Cz}, \mathrm{CPz}, \mathrm{P} 1, \mathrm{Pz}, \mathrm{P} 2, \mathrm{PO} 3, \mathrm{POz}, \mathrm{PO} 4$, according to international 10-20 system. The reference and ground electrodes were linked to the left and right mastoids, respectively.

\subsection{Experiment design}

Four healthy male subjects (aged 23-36 with average 31 , three right-handed and one left-handed) participated in the experiments. Each experiment started with a pause (approximately 90s) needed for the stabilization of the EEG signal. During this period, the EEG device transmits data but it is not processed. 
During recording, columns and rows of the matrix were highlighted in a random manner. The highlighting duration was $100 \mathrm{~ms}$, followed by a $100 \mathrm{~ms}$ of no highlighting. Each column and each row was flashed only once during one trial, so each trial consisted of 12 stimulus presentations.

As mentioned above, one trial is not enough for robust ERP detection; hence, we adopted the common practice of averaging the recordings over several trials before performing the classification of the (averaged) recordings. During recording, all 36 characters from the P300 matrix were presented to the subject. For each one of them, 10 trials were performed.

The subject was asked to count the number of highlightings of the desired character. The counting was used to maintain the subject's attention.

The recorded data were filtered in the $0.5-15 \mathrm{~Hz}$ frequency band, with a fourth order zero-phase digital Butterworth filter and properly cut into signal epochs. Each of these epochs consisted of $800 \mathrm{~ms}$ of recording, starting from the stimulus onset.

The data corresponding to the 18 first characters was used to train the classifier and the data corresponding to the 18 remaining characters was used to measure the performance of the classifier. Since we are interested in minimizing the number of trials needed for detection, we have built a series of classifiers on the training data, from single trial classification up to 10 trial classification (10fold averaging), and tested these classifiers on the test data averaged over the corresponding number of trials.

\section{Feature extraction}

Three types of features were used for training the classifier.

\subsection{Features based on downsampled signals}

We first selected features based on filtered and downsampled signals. Since the signals are recorded with a sampling frequency of $1000 \mathrm{~Hz}$, each $800 \mathrm{~ms}$ epoch consists of 800 data points. In order to reduce this number, epochs were "average downsampled" with a downsampling rate of 25 , meaning that the retained data points were averages over the nearest 25 data points. We obtained thus 32 points for each of the 8 channels and the $8 * 32=256$ corresponding values were selected as features for further classification.

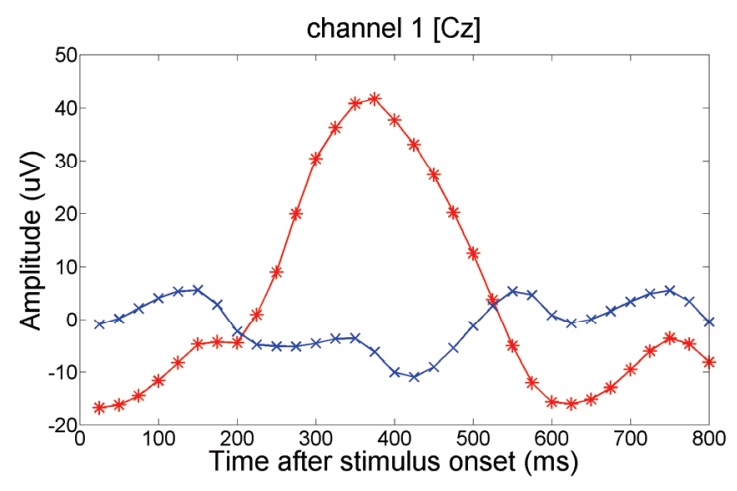

Figure 4: Grand average responses to target (stars) and non-target (crosses) stimuli at electrode position $\mathrm{Cz}$ for subject D.K. (filtered downsampled signals).

\subsection{Features based on Continuous Wavelet Transform}

The second type of features was obtained through the $\mathrm{t}$-CWT technique which is an ERP detection method based on the continuous wavelet transform and the Student's t-statistic [17]. For each channel, the CWT of each single trial of the training data is computed:

$$
W(s, t)=\frac{1}{\sqrt{s}} \int_{-\infty}^{\infty} f(\tau) \psi\left(\frac{\tau-t}{s}\right) d \tau
$$

with $\psi(t)=\frac{1}{\sqrt{2 \pi}}\left(1-t^{2}\right) e^{-t^{2} / 2} \quad$ (mexican hat wavelet)

In order to measure the points with the largest discriminatory power between different CWTs of the EEG responses to the target rows/columns and nontarget rows/columns, the Student's t-statistic is calculated at each point:

$$
t c w t(s, t)=\sqrt{\frac{N_{1} N_{2}}{N_{1}+N_{2}}} \frac{d(s, t)}{\sigma(s, t)}
$$

With $N_{1}$ the number of target responses, $N_{2}$ the number of non-target responses, $d(s, t)$ the difference average CWT between the 2 groups and $\sigma(s, t)$ the pooled variance.

Based on the training data, the 16 points $(s, t)$, with the largest absolute value of the Student's t-statistic, were selected for each channel, and the corresponding values of the CWT's were added as features to our classifier. Such a selection can be time consuming on the training data due to the fact that the CWTs are computed on a wide range of scales (s) and times ( $t$ ), and that the 
Student's t-statistics are calculated. However, the feature extraction on the test data can perfectly be performed in real-time since the selected points are already known (it only needs to compute 16 convolution products).
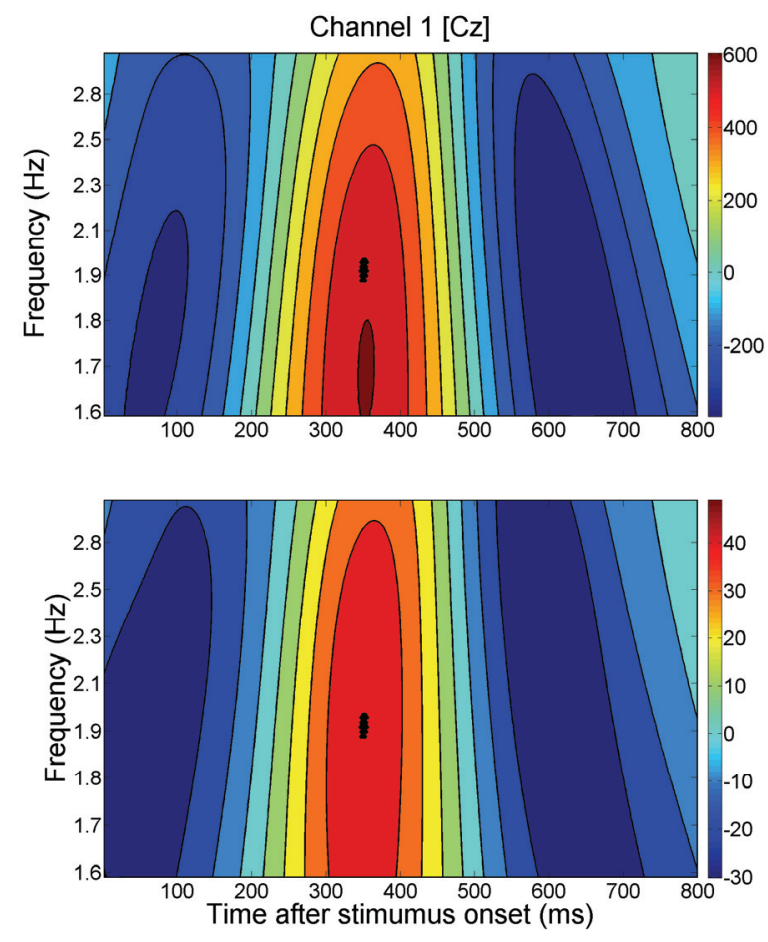

Figure 5: Grand average difference CWT (top) and corresponding Student's t-statistic (bottom) at electrode position $\mathrm{Cz}$ for subject D.K. The black triangles are the 16 selected points.

\subsection{Features based on Common Spatial Pattern}

The third and last type of features was obtained through the Common Spatial Pattern (CSP) technique [14]. This method has been originally used for the detection of abnormal EEG signals [18], and was successfully applied to the classification of movementrelated EEG signals [19].

The CSP is a technique of spatial filtering that finds the directions of optimal discriminability of two classes through variance. It maximizes the variance of one condition and at the same time minimizes the variance of the other condition.

Given the number of EEG channels $n$, the number of samples per channel $t$, the $n$ by $t$ matrix of EEG data (concatenated trials) of the first condition $P$ and the $n$ by $t$ matrix of EEG data (concatenated trials) of the second condition $Q$, the CSP technique computes the $n$ by $n$ matrix $W$ such that the normalized variances: $\operatorname{var}\left(W_{i} P\right)=$ $1-\operatorname{var}\left(W_{i} Q\right)$. Where $W_{i}$ represents the $i^{\text {th }}$ row of $W$.

By taking the rows of $W$ for which those variances are the closest to $0 / 1$, one obtains the directions of optimal discriminability through variance.

The matrix $W$ was build from the training set and the 4 most discriminative directions were kept. For each single trial $i$, given $S_{i}$ the $n$ by $t$ matrix of EEG data and $W$ the 4 by $n$ CSP matrix, the following four features were added to the classifier:

$$
f_{k}=\log \left(\frac{\operatorname{var}\left(W_{k} S\right)}{\sum_{i=1}^{4} \operatorname{var}\left(W_{i} S\right)}\right) \quad k=1, \ldots, 4
$$

\section{Classification and results}

The classifiers were trained on $2160 / \mathrm{n}$ data $(2160=18$ training characters $\times 12$ rows/columns flashed $\times 10$ trials per character and $\mathrm{n}$ the number of trials considered for averaging) and tested on $18 \times 12=216$ data. The dimension of the input space (number of features) was 388 .

To solve the classification problem, we explored several techniques; from very simple ones (Linear Discriminant Analysis, Fisher Linear Discriminant Analysis) to more complex ones (Radial Basis Function kernel based Support Vector Machines). We obtained the best performances by using a linear Least-Squares Support Vector Machine (LSSVM) [15].

A linear LSSVM finds the hyperplane in the feature space that best separates the data points of the two conditions (target stimulus and non-target stimulus) into two "half-planes" according to the following minimization problem (by use of the Lagrangian, a linear problem is solved in the Dual Space, see [15] for more details):

$$
[P]\left\{\begin{array}{l}
\min _{w, b, e} J_{p}(w, b), \text { where } J_{P}(w, b)=\frac{1}{2} w^{T} w+\gamma \sum_{k=1}^{N} e_{k}{ }^{2}, \\
\text { such that } y_{k}\left[w^{T} x_{k}+b\right]=1-e_{k}, k=1, \ldots, N
\end{array}\right.
$$

where $x_{k}$ corresponds to one data point in the feature space and $y_{k}$ is the associated output ( +1 for the responses to the target stimulus and -1 for the non-target stimulus), $w^{T} x+b=0$ is the equation of the separating hyperplane and $\gamma$ is the regularization parameter. 
In order to find the optimal value for the regularization parameter, the classifier was tuned on the normalized training features by a 5 -fold cross-validation with several starting values for $\gamma(0.001,0.02,0.1,0.5,1,2$ and 5$)$ using a grid-search procedure.

The classifier was used on the test data (to which the training normalization coefficients were applied) in the following way:

For each test character we have twelve points in the feature space (those features were extracted from the test signals averaged over the desired number of trials), six of them correspond to the responses to the flashing of the columns and the other six correspond to the responses to the flashing of the rows. In order to find the correct target letter we need to extract the row and column for which our classifier gives the best scores. Thus for each of those twelve points, the distance to the "LSSVM hyperplane" is calculated and for each of the six "row points" (or "column points"), we select the one that is the farthest away from the hyperplane in the target half-space. In the case that none of the six points would be laying in the target half-space, we select the one that is closest to the hyperplane (and thus to the target space).

We present here the performances of our classifier with respect to the number of trials used for averaging. The average performance over the subjects is compared with the results from the best P300 mind speller system we could find in the literature [20]. With only four averages, we reached an accuracy of $94.5 \%$ which would allow a subject to communicate a character in 9.6 seconds, instead of 22 seconds with 10 averages for an accuracy of $95.1 \%[20]$.

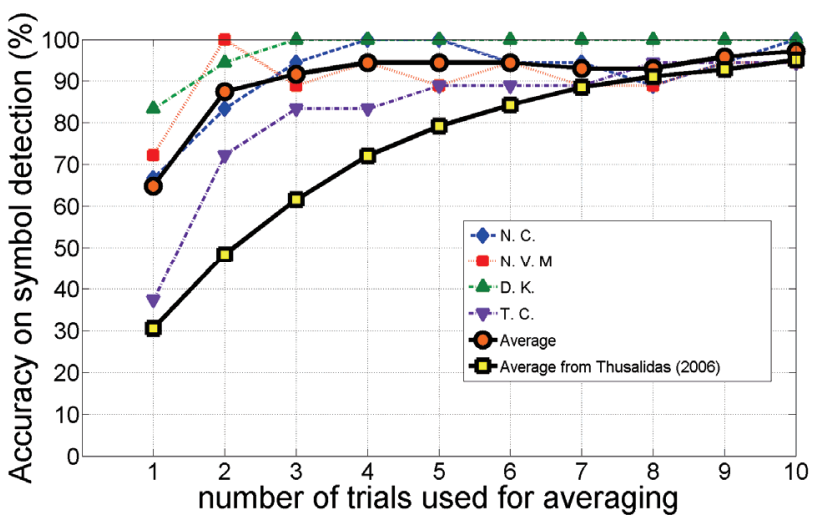

Figure 7: Accuracy of classification for different subjects as a function of the number of trials used in testing. Averaged result and result from [20] are also plotted.
Albeit the performance looks promising, more subjects need to be tested (the results from [20] are averaged over nine subjects). But the difference in performance between our study and the one presented in [20] seem to support our claim that the selected features and machine learning algorithm can be of great interest for the detection of ERPs in low signal-to-noise ratio EEGs and, in particular, for the P300 Mind Speller.

\section{Conclusion and outlook}

Our method has a computational drawback: the tuning of the LSSVM classifier is very time consuming and does not allow the test session to start immediately after the recording of the training data, which could be a problem for patients. But this can be remedied to a certain extend.

First, the stability over time of our set-up needs to be studied: ideally, the classifier does not need retraining before each new online typing session, so that we would have to train it only once. However, changes in experimental conditions (shifts in electrode positions, changes in impedance, etc...) may cause the classifier to be retrained before each session, but perhaps not from scratch, if the changes are minor, also given the superior performance of the trained classifier.

For example, one could decide to re-tune the classifier using as the starting value for the regularization parameter the value obtained in the initial training session, or even to skip this tuning part and retrain the classifier with the same value of the regularization parameter. Anyhow, by reducing the number of starting values for optimizing the regularization parameter, and by distributing the grid search algorithm onto parallel hardware, such as multiple cores, one could significantly reduce the training time.

Moreover, techniques other than cross-validation and grid-search can be used for tuning the LSSVM classifier: in [15] a method of Bayesian inference of parameters is proposed which proves to be less time consuming and can reach similar performances.

Finally, the addition of a spelling correction system such as T9, which is popular in SMS applications, can reduce the need to retrain the classifier since it can cope with extensive typing errors. It could also enable the subject to type faster common words or phrases.

To conclude, even though a lot of work is still ahead to achieve a robust P300 Mind Speller, that is also usable for patients, the current study shows that a subject can spell words more than twice as fast as the current state of the art. In addition, the use of a small wireless EEG device offers an improved wearability for the patient. 


\section{Acknowledgments}

$\mathrm{AC}$ and NVM are supported by the European Commission (IST-2004-027017). NC is supported by the European Commission (STREP-2002-016276). JAKS acknowledges support from research grants received from the Belgian Fund for Scientific Research - Flanders (G.0588.09). MMVH is supported by research grants received from the Excellence Financing program (EF 2005) and the CREA Financing program (CREA/07/027) of the K.U.Leuven, the Belgian Fund for Scientific Research - Flanders (G.0234.04 and G.0588.09), the Interuniversity Attraction Poles Programme - Belgian Science Policy (IUAP P5/04), the Flemish Regional Ministry of Education (Belgium) (GOA 2000/11), and the European Commission (STREP-2002-016276, IST-2004027017, and IST-2007-217077).

\section{References}

[1] Editorial Comment: Is this the bionic man? Nature 442(7099) (July 2006) 109.

[2] Editorial Comment: Sajda P., Müller K.R., and Shenoy K.V.: Brain-Computer Interfaces, IEEE Sig. Proc. Magazine, vol. 25, issue 1, pp 16-17, 2008.

[3] Pesaran, B., Musallam, S., Andersen, R.: Cognitive neural prosthetics. Current Biology 16(3) (2006) 77-80.

[4] Vidal, J.: Toward direct brain-computer communication. Annual review of Biophysics and Bioengineering 2(1) (1973) 157-180.

[5] Martinez P, Bakardjian H, and Cichochi A.: Fully online multicommand brain - computer interface with visual neurofeedback using SSVEP paradigm. Computational Intelligence and Neuroscience, vol. 2007,

[6] Ming Cheng, Xiaorong Gao, Shangkai Gao, Dingfeng $\mathrm{Xu}$, Design and implementation of a Brain-Computer Interface with high transfer rates, IEEE Trans. Biomed. Eng., vol. 11, pp 1181-1186, Oct. 2002.

[7] N. Birbaumer, A. Kubler, N. Ghanayim, T. Hinterberger, J. Perelmouter, J. Kaiser, I. Iversen, B. Kotchoubey, N. Neumann, and H. Flor, The thought translation device (TTD) for completely paralyzed patients, IEEE Transactions on Rehabilitation Engineering, vol. 8, no. 2, pp. 190-193, 2000.

[8] B. Blankertz, G. Dornhege, M. Krauledat, K.R. M"uller, and G. Curio, The non-invasive Berlin braincomputer interface: fast acquisition of effective performance in untrained subjects, NeuroImage, vol. 37, no. 2, pp. 539-550, 2007.

[9] J.R. Wolpaw, D.J. McFarland, and T.M. Vaughan, Brain-computer interface research at the Wadsworth
Center, IEEE Transactions on Rehabilitation Engineering, vol. 8, no. 2, pp. 222-226, 2000.

[10] S.J. Luck, An introduction to the event-related potential technique, MIT Press Cambridge, MA, 2005.

[11] W.S. Pritchard, Psychophysiology of P300, Psychological Bulletin, vol. 89, no. 3, pp. 506, 1981.

[12] L.A. Farwell and E. Donchin, Talking off the top of your head: toward a mental prosthesis utilizing event related brain potentials, Electroencephalography and clinical Neurophysiology, vol. 70, no. 6, pp. 510-523, 1988.

[13] Samar, V. J., Bopardikar, A., Raghuveer, M. K., \& Swartz, K. 1999. Wavelet analysis of neuroelectric waveforms: A conceptual tutorial. Brain and Language, $66,7-60$.

[14] H. Ramoser, J. Müller-Gerking, and G. Pfurtscheller, Optimal spatial filtering of single trial EEG during imagined hand movement, IEEE Transactions on Rehabilitation Engineering, vol. 8, no. 4, pp. 441-446, 2000.

[15] Suykens J.A.K., Van Gestel T., De Brabanter J., De Moor B., Vandewalle J. (2002), Least Squares Support Vector Machines, World Scientific, Singapore.

[16] Yazicioglu, R., Merken, P., Puers, R., Van Hoof, C.: Low-power low-noise 8-channel EEG front-end ASIC for ambulatory acquisition systems. In: Solid-State Circuits Conference, 2006. ESSCIRC 2006. Proceedings of the 32nd European. (2006) 247-250.

[17] Bostanov, V. and B. Kotchoubey (2006), The tCWT: a new ERP detection and quantification method based on the continuous wavelet transform and Student's t-statistics, Clinical Neurophysiology, 117, 2627-2644.

[18] Z. J. Koles, The quantitative extraction and topographic mapping of the abnormal components in the clinical EEG, Electroenc. Clin. Neurophys. vol. 79, pp. 440-447, 1991.

[19] J. Müller-Gerking, G. Pfurtscheller, and H. Flyvbjerg, Designing optimal spatial filters for single-trial EEG classification in a movement task, Electroenc. Clin. Neurophys, vol. 110, no. 5, pp. 787-798, 1999.

[20] Thulasidas, M., Guan, C., Wu, J.: Robust classification of EEG signal for brain computer interface. IEEE Transactions on Neural Systems and Rehabilitation Engineering 14(1) (2006) 24-29. 